\title{
On Spheroidal Harmonics and Allied Functions.
}

\author{
By G. B. JefFery.
}

(Received 19th May 1915. Read 11th June 1915).

In a paper recently read before this Society, ${ }^{*}$ Mr E. Blades obtained a general formula for spheroidal harmonics in the form of the general solution of Laplace's equation given by Professor Whittaker,

$$
\int_{0}^{2 \pi} f(x \cos t+y \sin t+i z, t) d t
$$

If spheroidal coordinates $r, \theta, \phi$ are defined by

$$
\begin{aligned}
& x=c \sqrt{r^{2}+1} \sin \theta \cos \phi \\
& y=c \sqrt{r^{2}+1} \sin \theta \sin \phi \\
& z=c r \cos \theta
\end{aligned}
$$

the result obtained is

$$
\begin{aligned}
& \frac{1}{2 \pi} \int_{0}^{2 \pi} P_{n}\left(\frac{j \cos t+y \sin t+i z}{c}\right) \sin m t d t \\
& =\frac{(n-m) !}{(n+m) !} P_{n}^{m}(i r) P_{n}^{m}(\cos \theta) \underset{\cos }{\sin } m \phi .
\end{aligned}
$$

This formula gives, for real and pure imaginary values of $r$ respectively, all the oblate and problate spheroidal harmonics which are finite in the finite region. It does not include the harmonics which vanish at infinity, which are, perhaps, from the point of view of physical application, the more interesting class. These can, however, be obtained by the same method as that employed by Mr Blades.

The addition theorem for Legendre functions of the second kind was given by Heine. $\dagger$ After making some changes in the

* Proc. Edin. Math. Soc., Vol. XXXIII. (Part I.), 1915, p. 65.

+ Kugelfunctionen 1, p. 333. 
numerical factors in order to conform to the now accepted notation for the associated Legendre functions, ${ }^{*}$ the theorem may be written

$Q_{n}\left(\cos \theta \cos \theta^{\prime}+\sin \theta \sin \theta^{\prime} \cos (\phi-c)\right)=P_{n}(\cos \theta) Q_{n}\left(\cos \theta^{\prime}\right)$

$$
+2 \sum_{m=1}^{\infty} \frac{(n-m) !}{(n+m) !} P_{n}^{m}(\cos \theta) Q_{n}^{m}\left(\cos \theta^{\prime}\right) \cos m(\phi-c) \text {. }
$$

Multiplying by $\sin m t$, and integrating with respect to $t$ from 0 to $2 \pi$, we have

$$
\begin{aligned}
\frac{1}{2 \pi} \int_{0}^{2 \pi} Q_{n}\left(\cos \theta \cos \theta^{\prime}+\sin \theta \sin \theta^{\prime} \cos (\phi-t)\right) & \sin _{\cos }^{\sin } m t d t \\
& =\frac{(n-m) !}{(n+m) !} P_{n}^{m}(\cos \theta) Q_{n}^{m}\left(\cos \theta^{\prime}\right) \cos _{\cos }^{\sin \phi}
\end{aligned}
$$

Put $\cos \theta=i r$, and this may be written

$$
\begin{aligned}
\frac{1}{2 \pi} \int_{0}^{2 \pi} Q_{n}\left(\frac{x \cos t+y \sin t+i z}{c}\right) & \sin m t d t \\
= & \frac{(n-m) !}{(n+m) !} P_{n}^{m}(\cos \theta) Q_{n}^{m}(i r) \frac{\sin }{\cos } m \phi .
\end{aligned}
$$

If $r$ is real, these solutions become infinite on the circle $z=0, x^{2}+y^{2}=c^{2}$; if $r$ is a pure imaginary, they become infinite on that part of the axis of $z$ between the points $z= \pm c$; in either case they have infinities inside every spheroid of the family $r=$ const. On the other hand they tend to zero for large values of $r$, i.e. at points at a great distance from the origin.

The only other class of spheroidal harmonic consists of the product of two Legendre functions, both of which are of the second kind. These solutions become infinite either at all points on the axis of $z$ or at all points on the plane $z=0$. These solutions have not as yet been used to any great extent in mathematical physics, but, from the point of view of the theory of the solution of Laplace's equation, it would be interesting to see the form of the function $f$ in Whittaker's solution which gives rise to them.

\footnotetext{
* e.g. That employed in Whittaker's Modern Analysis.
} 
The analogous theorems for Bessel functions also give solutions of Laplace's equation. Neuman's generalised addition theorem may be written*

$$
\begin{aligned}
& J_{0} \sqrt{\left.b^{2}-2 b c \cos (\phi-t)+c^{2}\right)} \\
& \quad=J_{0}(b) J_{0}(c)+2 \sum_{1}^{\infty} J_{m}(b) J_{m}(c) \cos m(\phi-t)
\end{aligned}
$$

and there is a corresponding theorem for functions of the second kind

$$
\begin{aligned}
& Y_{0}\left(\sqrt{b^{2}-2 b c \cos (\phi-t)+c^{2}}\right) \\
& \quad=Y_{0}(b) J_{0}(c)+2 \sum_{1}^{\infty} Y_{m}(b) J_{m}(c) \cos m(\phi-t) .
\end{aligned}
$$

Multiplying by $\sin _{\cos }^{\sin } m$ and integrating from 0 to $2 \pi$ we have

$$
\begin{aligned}
\frac{1}{2 \pi} \int_{0}^{2 \pi} J_{0}\left(\sqrt{\left.b^{2}-2 b c \cos (\phi-t)+c^{2}\right)}\right. & \frac{\sin }{\cos m t d t} \\
& =J_{m}(b) J_{m}(c) \cos _{\cos }^{\sin } m
\end{aligned}
$$

and

$$
\begin{aligned}
\frac{1}{2 \pi} \int_{0}^{2 \pi} Y_{0}\left(\sqrt{\left.b^{2}-2 b c \cos (\phi-t)+c^{2}\right)}\right. & \frac{\sin }{\cos } m t d t \\
& =Y_{m}(b) J_{m}(c) \sin _{\cos }^{\sin } m .
\end{aligned}
$$

Take coordinates $\xi, \eta, \phi$ defined by

$$
\begin{aligned}
& x=2 \xi \eta \cos \phi \\
& y=2 \xi \eta \sin \phi \\
& z=\xi^{2}-\eta^{2} .
\end{aligned}
$$

*Gray and Mathew's Bessel's Functions, p. 27 and p. 92. We have followed the notation there used for the second solution of Bessel's equation, but it is clear that in this case $Y_{m}$ may be taken as any lincar combination of the two solutions in which the coefficients are independent of $m$. 
'The surfaces $\xi, \eta=$ const. are a double orthogonal system of confocal paraboloids of revolution, having for a common axis the axis of $z$. Writing $b=n \xi, c=i n \eta$, we have

$$
\begin{aligned}
\frac{1}{2 \pi} \int_{0}^{2 \pi} J_{0}\left(n e^{-i \frac{\pi}{4}} \sqrt{x \cos t+y \sin t+i z)} \frac{\sin }{\cos m t d t}\right. & =J_{m}(n \xi) J_{m}(i n \eta) \frac{\sin }{\cos m \phi}
\end{aligned}
$$

and

$$
\begin{aligned}
\frac{1}{2 \pi} \int_{0}^{2 \pi} Y_{0}\left(n e^{-i \frac{\pi}{4}} \sqrt{x \cos t+y \sin t+i z)}\right. & \sin m t d t \\
& =Y_{m}(n \xi) J_{m}(i n \eta) \stackrel{\cos m \phi .}{\cos m}
\end{aligned}
$$

These solutions of Laplace's equation are suitable for the investigation of problems in which the boundary conditions are given over paraboloids of revolution. The former remains finite when either $\xi$ or $\eta$ vanishes, i.e. at all points on the axis of $z$. This then is the solution which must be employed for the interior of a paraboloid of revolution. The second solution becomes infinite when $\xi=0$, and therefore it may be employed for the field exterior to one of the paraboloids, $\xi=$ const. The first solution is also finite throughout the region exterior to this paraboloid, and hence in this case we must employ a linear combination of the two solutions determined by the conditions at infinity. 